\title{
Massive stars at (very) high energies: $\gamma$-ray binaries
}

\author{
Guillaume Dubus and Benoît Cerutti
}

\author{
Laboratoire d'Astrophysique de Grenoble, UMR 5571 Université J. Fourier \& CNRS, France \\ email: gdubus@obs.ujf-grenoble.fr
}

\begin{abstract}
MeV}$. They are associated with $\mathrm{O}$ or Be stars in orbit with a compact object, possibly a young pulsar. Much like colliding wind binaries, the pulsar generates a relativistic wind that interacts with the stellar wind. The result is non-thermal emission from radio to very high energy $\gamma$-rays. The wind, radiation and magnetic field of the massive star play a major role in the dynamics and radiative output of the system. They are particularly important to understand the high energy physics at work. Inversely, $\gamma$-ray binaries offer novel probes of stellar winds and insights into the fate of $\mathrm{O} / \mathrm{B}$ binaries.
\end{abstract}

Keywords. radiation mechanisms: nonthermal, pulsars: general, stars: winds, outflows, gamma rays: observations, gamma rays: theory, X-rays: binaries

High energy (HE) $\gamma$-rays have energies above $100 \mathrm{MeV}$, very high energy $\gamma$-rays (VHE) are above $100 \mathrm{GeV}$. This definition has an instrumental origin: both HE and VHE $\gamma$-rays are stopped in the upper atmosphere but the Cherenkov light from the electromagnetic showers initiated by VHE $\gamma$-rays can be detected from the ground using large telescopes with fast detectors. Our knowledge of the HE and VHE sky is increasing rapidly thanks to a successful collection of new instruments. In HE $\gamma$-rays the Fermi $\gamma$-ray space telescope has been operating for two years. Its first catalog, published earlier this year, contains 1500 sources when 250 were previously known. In VHE $\gamma$-rays, more than 80 sources have been discovered by the arrays of Cherenkov telescopes that have now been operating for 5-7 years (HESS, VERITAS, MAGIC): only a handful of confirmed sources were known in 2003. This bonanza has started to impact many fields of astrophysics, including massive stars, and will hopefully continue in the future with projects like the Cherenkov Telescope Array (CTA). Although this paper will be concerned with $\gamma$-ray binaries, the recent discoveries of HE emission from stellar clusters like 30 Dor (Abdo et al. 2010b) or from the direction of $\eta$ Car are also susceptible to be of interest to the massive star community.

\section{Binaries at high energies}

There is, at present $(7 / 2010)$, four confirmed binaries emitting HE or VHE $\gamma$-rays: PSR B1259-63, LS 5039, LS I+61 303 and Cyg X-3 (Holder 2009). Three other candidates can be added: HESS J0632+057 (confirmed, variable, VHE and radio source coincident with the Be star MWC148 but without evidence yet for a compact companion), Cygnus X1 (well-known black hole candidate orbiting an $\mathrm{O}$ star but the VHE $\gamma$-ray detection during a brief flare was marginal and has not been independently confirmed; AGILE has also reported marginal HE detections not confirmed by Fermi) and the latest addition, V407 Cyg, a symbiotic system (white dwarf orbiting a red giant) where HE $\gamma$-rays were detected during a nova outburst (Abdo et al. 2010a). V407 Cyg is a nice illustration of the surprises the HE sky has in store for us. 
V407 Cyg aside, the other systems are all high mass X-ray binaries where a compact object, neutron star or black hole, orbits a Be or an O star. The four confirmed binaries all show modulations at the orbital period in $\gamma$-rays. In LS 5039, the orbital period can be determined independently from the VHE modulation with a precision comparable to that obtained with radial velocities. Optical spectroscopy gives access to the orbital and stellar parameters (see McSwain, these proceedings). It remains the essential first step from which stems all understanding of these systems.

Microquasars. The binaries can be set in two groups. The Cygnus sources are both well-known accreting sources where material from the massive star falls onto the compact object, releasing gravitational energy. The compact object in Cyg X-3 orbits a Wolf-Rayet star every 4.8 hours. Accretion is accompanied by the occasional ejection. Radio flares and $\mathrm{X}$-ray state changes are then seen. The radio emission has been resolved into relativistic jets. $\gamma$-ray emission from Cyg X-3 has been detected only during soft X-rays states and high flux radio states, that is when the relativistic jet is known to be present (The Fermi LAT Collaboration, Abdo et al. 2009). This implies $\gamma$-ray emission is related in some way to the accretion-ejection physics. This holds great promise to further our understanding of the link between non-thermal emission and accretion/ejection, processes that are also at work in AGNs and $\gamma$-ray bursts. The detected $\gamma$-ray spectral luminosity represents $<10 \%$ of the X-ray luminosity.

$\gamma$-ray binaries. Emission above 1-10 $\mathrm{MeV}$ dominates the radiative output in those systems, hence their name (Dubus 2006). Their X-ray luminosities are moderate $\left(10^{33}-\right.$ $\left.10^{34} \mathrm{erg} \mathrm{s}^{-1}\right)$, their optical spectra are dominated by the massive star. All are radio sources and that is rare amongst high-mass X-ray binaries. However, it is really in $\gamma$ rays that the unusual character of these objects is revealed and sensitive Galactic Plane surveys carried out by CTA in the future should discover more examples.

\section{2. $\gamma$-ray binaries as binary pulsar wind nebulae}

Similarities in the spectra and behaviour of $\gamma$-ray binaries have led to the suggestion that all may belong to a class of systems different from the accreting microquasars. The key is PSR B1259-63, a 48 ms radio pulsar in a 3.5 year orbit around a Be star. Timing of the radio pulses enables a very good determination of the orbit, but also to measure the spindown rate of the pulsar. The corresponding rotational power $\dot{E}$ that is lost is $810^{35}$ $\mathrm{erg} \mathrm{s}^{-1}$, the inferred magnetic field is $\approx 310^{11} \mathrm{G}$ and the spindown timescale is $\approx 310^{5}$ years. Radio flaring is associated with the crossing of the Be disc. Radio flares are seen in LS I+61 303 (the orbital ephemeris is derived from them) and radio variability has been detected in HESS J0632+057 (Skilton et al. 2009); both have Be companions.

Pulsar winds. The magnetic field dominates the immediate environment of the neutron star. Particles accelerated in the magnetosphere are thought to be responsible for the pulsed $\gamma$-ray emission seen now from $\approx 60$ pulsars by Fermi (pulsars are the dominant HE population in the Galactic Plane). Particles on the field lines cannot co-rotate with the neutron star beyond the light cylinder radius $\left(c P_{\mathrm{ns}} / 2 \pi\right)$, where their angular speed attains $c$. Magnetic field lines open up and a relativistic wind is launched, carrying away the rotational energy of the neutron star. Pulsar winds interacting with the surrounding material (supernova remnant, ISM) create pulsar wind nebulae: the canonical PWN is the Crab nebula but dozens have now been detected in VHE $\gamma$-rays (here also PWN are the dominant VHE population in the Galactic Plane). In PSR B1259-63 the interaction occurs with the stellar wind or the disc of the Be star. The other established pulsars with B/Be companions are PSR J1740-3052 and PSR J0045-7319. Unfortunately, they 
are too far to be detectable in $\gamma$ rays $(\approx 11 \mathrm{kpc}$ and SMC when PSR B1259-63 and the other $\gamma$-ray binaries are 2 to $3 \mathrm{kpc}$ away).

What about accretion? Neutron stars in orbit around massive companions can accrete matter from the stellar wind. The Bondi-Hoyle accretion rate onto a compact object moving with speed $v$ in a medium with density $\rho$ is $\dot{M}_{\mathrm{b}}=\pi R_{\mathrm{b}}^{2} \rho v$, where $R_{\mathrm{b}}=2 G M / v^{2}$ is the capture radius. SPH simulations show good agreement with this estimate (Okazaki, these proceedings). The pressure from accretion grows like $R^{-5 / 2}$ where $R$ is the distance to the neutron star, whereas the pulsar pressure increases like $R^{-2}$. Accretion will be held off if the pulsar wind pressure is greater than the accretion pressure at the capture radius. This requires $\dot{E}>4 \dot{M}_{\mathrm{b}} v c$ or $P_{\mathrm{ns}} \leqslant 0.23 \dot{M}_{15}^{-1 / 4} B_{12}^{1 / 2} \mathrm{~s}$. The Bondi accretion rate at 0.1 a.u. in a $1000 \mathrm{~km} / \mathrm{s}, 10^{-6} \mathrm{M}_{\odot} \mathrm{yr}^{-1}$ wind (LS 5039) is about $10^{15} \mathrm{~g} \mathrm{~s}^{-1}$. It can increase dramatically in a Be disc. Still, a fast-spinning neutron star with a strong field, i.e. a young pulsar, can prevent accretion even at Eddington rates (Dubus 2006). As the pulsar spins down on a timescale of a few $10^{5}$ years the pressure from its wind will decrease until accretion pushes its way to the neutron star and a high-mass X-ray binary turns on. $\gamma$-ray binaries are a short lived phase following the SN explosion and preceding the HMXB phase, as anticipated in population synthesis of HMXBs that predict fewer than 100 such systems in our Galaxy.

\section{The radiation field of the massive star}

The $\gamma$-ray orbital modulation can happen either because the VHE particles radiate in a phase-dependent manner or because the injection of those particles is phasedependent. Radiative processes are well known, unlike particle acceleration processes, so these systems offer a unique chance to separate the two. Understanding the orbital phase-dependent effects of radiative processes carries the hope of constraining where and how the VHE particles get their energy. Leptonic models assume these particles are electrons (and positrons). The main radiative processes are synchrotron emission and inverse Compton radiation. Synchrotron radiation cannot produce radiation above $\approx 100$ $\mathrm{MeV}$. Beyond this limit, synchrotron losses are so quick that the process through which the electron originally gained energy would have to occur on a fraction of the time it takes to turn around the field line, requiring exotic acceleration mechanisms. Therefore, $\gamma$-ray radiation is very likely to be inverse Compton (IC) radiation. Models based on high-energy protons (hadronic models) radiating via $p p$ or $p \gamma$ interactions have not been much pursued as they require more energy and do not easily explain the modulations.

Inverse Compton modulation The most abundant seed photons are by far those of the massive star (about $10^{14}$ photons $\mathrm{cm}^{-3}$ at the location of the compact object in LS 5039). IC interactions on this external source readily produces an orbital modulation: there is a maximum when the seed photons are backscattered towards the observer (superior conjunction) and a minimum when they are forward-scattered (inferior conjunction). In addition, there can be spectral changes as the interaction occurs in the Klein-Nishina or Thompson IC regime depending on angle (Dubus et al. 2008).

Pair production Once emitted, VHE $\gamma$ rays can have enough energy to create $e^{+} e^{-}$ pairs through their interaction with photons from the star $\left(\gamma+\gamma \rightarrow e^{+}+e^{-}\right)$. The threshold for this reaction is $\epsilon_{\gamma} \epsilon_{\star} \geqslant 2\left(m_{e} c^{2}\right)^{2} /(1+\cos \psi)$ where $\psi$ is the angle between the two photons. For a stellar temperature of $40,000 \mathrm{~K}\left(\epsilon_{\star} \approx 9 \mathrm{eV}\right)$, this happens when the energy of the $\gamma$ ray $\epsilon_{\gamma} \geqslant 30 \mathrm{GeV}$. The cross-section is of order of the Thompson crosssection with maximum around a few $100 \mathrm{GeV}$, in the range of ground-based Cherenkov arrays. Because of the angle dependence, a constant source of VHE $\gamma$-rays orbiting the 

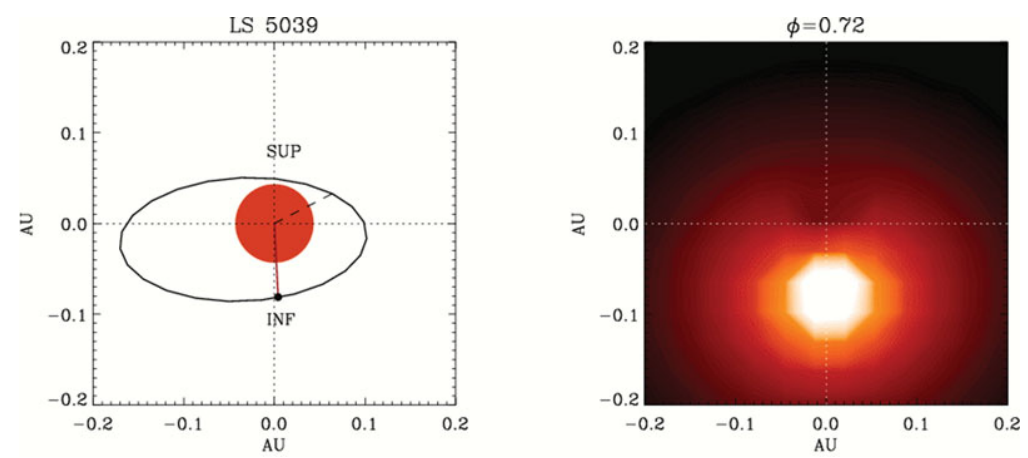

Figure 1. Map of the emission above $100 \mathrm{GeV}$ close to inferior conjunction in LS 5039. This includes inverse Compton emission, pair production and subsequent 3D cascading.

massive star will be modulated by absorption on stellar. Maximum absorption occurs when the two photons interact head-on and minimum when this is tail-on. The absorption maximum will therefore be at superior conjunction and the minimum at inferior conjunction.

$\underline{\text { Cascade }}$ The pairs created when $\gamma$ rays are 'absorbed' radiate. If they radiate VHE IC emission, the $\gamma$ rays (of slightly less energy) wills generate new pairs until the energy drops below the threshold for pair production. The geometry and the magnetic field control this cascade emission (Cerutti et al. 2010). If the magnetic field is strong the pairs will preferentially radiate synchrotron instead of IC and the cascade process will be stopped. If the magnetic field is very weak then the pairs propagate along the path of the initial $\gamma$ ray instead of being isotropized (Cerutti et al. 2009).

Here, unlike in GRBs or AGNs, the radiation field is set by the massive star. Together with the constrained geometry from the binary orbit, this allows detailed comparisons to be made between models and data. All the processes are at work in LS 5039: in HE there is an IC modulation, modified at VHE by pair production. At superior conjunction, the absorption is less important than expected, suggesting cascading: this requires the magnetic field to be $\leqslant 6 \mathrm{G}$ in the VHE emitting region. The $\mathrm{O}$ star should not have a strong magnetic field. Fermi observations of LS 5039 and LS I+61 303 show power law spectra with exponential cutoffs at a few GeV (akin to the Fermi pulsars), implying the particle population radiating $\mathrm{HE} \gamma$ rays is different from the one radiating VHE $\gamma$ rays. In the Be systems the phases of peak emission are difficult to explain with a purely radiative model and other effects are suspected. The interaction with the Be disc can lead to strong orbital variations in particle injection, escape or adiabatic cooling (see also van Soelen, these proceedings). $\gamma$-ray binaries are a window into particle acceleration under varying, but controlled, conditions.

\section{The stellar wind of the massive star}

There is now a reasonable understanding of the radiative processes at work in $\gamma$-ray binaries and their dependence with orbital phase (Bosch-Ramon \& Khangulyan 2009). There is much less understanding of the dynamics of the interaction between the pulsar wind and the stellar wind. This is accessible through different means.

Absorption in the stellar wind can affect radio and X-ray emission. The free free optical depth at $1 \mathrm{GHz}$ at a distance $d$ from the star is $\tau \approx 3 \cdot 10^{4} \dot{M}_{\mathrm{w}, 7}^{2} v_{w, 2000}^{-2} T_{4}^{-3 / 2} \nu_{\mathrm{GHz}}^{-2} d_{0.1}^{-3}$ for a $10^{-7} \mathrm{M}_{\odot} \mathrm{yr}^{-1}$ coasting wind with a speed $2000 \mathrm{~km} \mathrm{~s}^{-1}$ and temperature $10^{4} \mathrm{~K}$; $d$ is $0.1 \mathrm{AU}$, on the order of the orbital separation in LS 5039 and LS I+61 303. This 
makes it a priori impossible to detect a radio pulsar in these tight systems. PSR B1259-63 has a wider orbit but the radio pulsations are eclipsed for more than a month around periastron. The study of the eclipse show the absorption is best explained as due to the Be disc tilted with respect to the orbital plane Melatos et al. (1995). The absorption column $N_{H}$ in X-rays could also probe the wind interface. There is an orbital modulation of the X-rays from LS 5039 but the observed $N_{H}$ stays constant. This severely constraints the size of the X-ray source or the stellar wind density: the X-ray source should be $\geqslant 3 R_{\star}$ if the stellar wind mass loss rate is $10^{-7} \mathrm{M}_{\odot} \mathrm{yr}^{-1}$ (Szostek \& Dubus, submitted).

Variability in the stellar wind can also affect the $\gamma$-ray emission. One link is the radio outbursts of PSR B1259-63 (and the other $\gamma$-ray binaries with Be companions ?) that are thought to be related to the passage of the pulsar through the Be disc. There is evidence for a long term modulation on a 4 year timescale from the ephemerides of radio outburst in LS I+61 303 as well as from optical lines (Zamanov et al. 1999). Be disc precession might be the cause. The orbit-averaged HE $\gamma$-ray emission has recently been observed to rise in Fermi, at a time in the 4 year cycle when the $\mathrm{H} \alpha \mathrm{EW}$ and $\Delta V$ is large - suggesting a large Be disc. However, contemporary optical studies shown at this meeting did not report anything unusual. X-ray variability on timescales of minutes has also been reported in LS I +61 303. This has yet to be understood: possibilities include inhomogeneities in the stellar wind/Be disc leading to fluctuations in the wind interface, instabilities at the interface, accretion events or some unrelated source in the field-of-view. Magnetar-like activity has also been reported from LS I+61 303. The IR-optical band gives access to the stellar wind or Be disc and campaigns should include such observations to shed light on these issues.

Morphology of the pulsar wind - stellar wind interaction is accessible via radio observations. VLBI observations of LS I+61 303 (Dhawan et al. 2006) and LS 5039 (Ribo et al. 2008) show resolved, collimated radio emission on scales of milli-arcseconds (a.u. scales; unfortunately there is no data available for PSR B1259-63). These initially suggested relativistic jets as observed in Cygnus X-3 and other microquasars. However, the position angle of this emission changes with orbital phase. Furthermore, the changes in morphology are reproducible from orbit to orbit. Whereas this is very difficult to explain with a microquasar jet, whose orientation is not expected to change during one orbit, this is rather natural to expect if a pulsar wind is collimated into a comet-like trail by a strong stellar wind. Whether this happens depends on the ratio of wind momentum fluxes, as in colliding wind binaries (Bogovalov et al. 2008). The large scale expectation for isotropic winds is an Archimedean spiral with a step $v_{w} P_{\text {orb }}$ where $v_{w}$ is the terminal speed of the strongest wind (Lamberts, these proc.). What happens when the interaction is with a Be disc is unclear (but see Owocki, Okazaki, these proceedings). Numerical simulations using relativistic MHD are required to obtain the evolution of the magnetic field and speed in the shocked region together with the large scale morphology in order to reproduce the VLBI radio maps.

\section{Conclusion: massive stars in extreme environments}

More interaction is required between the $\mathrm{HE}$ and massive star communities. Many of the techniques used in colliding wind binaries can be applied to $\gamma$-ray binaries. The conditions in the wind and constraints on dynamics derived from optical spectra need to be looked at carefully (McSwain, these proceedings). Clumping may have noticeable effects although supergiant fast X-ray transients (a class of HMXBs) are probably better probes for this than $\gamma$-ray binaries. The massive star may be substantially modified by this extreme environment. For instance, calculations show the temperature in the wind 
may be increased to $10^{5} \mathrm{~K}$ by the $\gamma$-rays (Zdziarski et al. 2010): what are the consequences on wind acceleration? If the pulsar wind is strong it may impact directly onto the surface of its companion (as in black widow pulsars, old recycled ms pulsars with very low mass companions): what are the observable consequences, a dearth of UV lines at some phases?

\section{Acknowledgements}

We thank the OC for the smooth organization of the conference. This work was supported by the European Community via contract ERC-StG-200911.

\section{References}

Abdo, A. A., Ackermann, M., Ajello, M., Atwood, W. B. et al. 2010a, Science, 329, 817

Abdo, A. A., Ackermann, M., Ajello, M., Atwood, W. B. et al. 2010b, A\&\&A, 512, A7

The Fermi LAT Collaboration, Abdo, A. A., Ackermann, M., Ajello, M. et al. 2009, Science, 326, 1512

Bogovalov, S. V., Khangulyan, D. V., Koldoba, A. V., Ustyugova, G. V. et al. 2008, MNRAS, 387,63

Bosch-Ramon, V. \& Khangulyan, D. 2009, International Journal of Modern Physics D, 18, 347

Cerutti, B., Dubus, G., \& Henri, G. 2009, A\&A, 507, 1217

Cerutti, B., Malzac, J., Dubus, G., \& Henri, G. 2010, A\& A, 519A, 81

Dhawan, V., Mioduszewski, A., \& Rupen, M. 2006, in: VI Microquasar Workshop: Microquasars and Beyond, p. 52

Dubus, G. 2006, A\&A, 456, 801

Dubus, G., Cerutti, B., \& Henri, G. 2008, A\& $A, 477,691$

Holder, J. 2009, ArXiv e-prints 0912.4781

Melatos, A., Johnston, S., \& Melrose, D. B. 1995, MNRAS, 275, 381

Ribó, M., Paredes, J. M., Moldón, J., Martí, J. et al. 2008, A\&A, 481, 17

Skilton, J. L., Pandey-Pommier, M., Hinton, J. A., Cheung, C. C. et al. 2009, MNRAS, 399, 317

Zamanov, R. K., Martí, J., Paredes, J. M., Fabregat, J. et al. 1999, A\&̇A, 351, 543

Zdziarski, A. A., Neronov, A., \& Chernyakova, M. 2010, MNRAS, 403, 1873

\section{Discussion}

MCSWAIN: I just wanted to comment on the properties of the Be star in HESS J0632+057. The Be star has a temperature of $30,000 \mathrm{~K}$ and $\log g$ about 4.0. We have place constraints on the orbital period and find that it cannot be less than 100 days. These results should appear soon in a paper by Aragona et al.

Dubus: This is good to know, a long period could explain the VHE variability. Spectroscopic studies are crucial to understand what is going on.

OKASAKI: You mentioned the possibility that a large Be disc eclipses a radio pulsar so that the pulsar is not observed in systems other than PSR B1259-63. However, the Be disc can't be that big if the compact object is a radio pulsar with relativistic wind. Therefore, if there is a pulsar in these systems, it should be detected.

DuBus: The other systems have tighter orbits than PSR B1259-63. The radio pulsar is eclipsed in PSR B1259-63 when it goes round periastron: it is then at a distance from its Be companion that is still greater than the maximum separation in the other systems. Therefore, I don't think the environment will be less dense than for PSR B1259-63 even if the Be disc is not as large. Calculations (Dubus 2006, Zdziarski et al. 2010) show the free-free opacity is very large at $\mathrm{GHz}$ frequencies. It will be useful if you predict that there are phases with significantly less absorption. 